\title{
Characterization of 3D printed sheets using multi-frequency scanning impedance microscopy
}

\author{
Martijn Schouten and Gijs Krijnen \\ Robotics And Mechatronics group, University of Twente, Enschede, The Netherlands \\ Email: m.schouten@utwente.nl
}

口

\begin{abstract}
In this work we present a multi-frequency scanning impedance microscopy technique suitable for 3D printed structures. In this technique, a ball-head pogo pin is used to measure the voltage distribution in $3 \mathrm{D}$ printed samples by rolling it over the sample. It is shown that this technique can be used to measure the complex electric field distribution at varying frequencies in a single layer 3D printed conductor.
\end{abstract}

Keywords - $3 D$ printing, additive manufacturing, anisotropic, non-uniform, conductor, multi-frequency, scanning impedance microscopy, electrical impedance scanning

\section{INTRODUCTION}

Multi-material 3D printing is an interesting technology for the fabrication of 3D printed sensors because of customization possibilities and because it enables the fabrication of complex systems [1], [2]. However, due to the nature of additive manufacturing, the conductivity of printed conductors can be non-uniform and anisotropic [3], [4]. The conductivity of sheets of material has been characterized using voltage contrast scanning electron microscopy and (lock-in) infrared thermography [5], [6]. While these methods give a qualitative understanding of the potential distribution it is challenging to obtain a quantitative result.

In this paper, we propose a method similar to impedance scanning microscopy [7] and electrical impedance scanning [8], but adjusted to be suitable for 3D printed sensors. In this method, a multi-frequency current is sent through a $3 \mathrm{~d}$ printed sample mounted on the bed of a 3D printer. Next, a springloaded ball is rolled over the sample in a scanning pattern to measure the voltage distribution induced by this current.

It will be shown that using this method it is possible to quantitatively measure the real and imaginary electric field inside single layer 3D printed conductors for multiple frequencies.

\section{MODEL}

During the multi-frequency impedance scanning microscopy method the voltage distribution in the sample will be measured. This voltage distribution $\phi$ can be used to obtain the 2D electric field inside the structure $\vec{E}$ through [9]

This work was developed within the Wearable Robotics programme, funded by the Dutch Research Council (NWO).

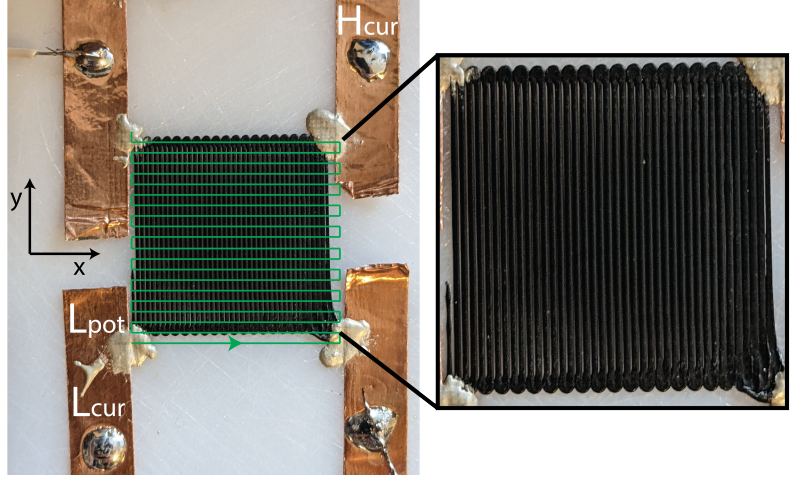

Fig. 1. A picture of the used sample.

$$
\vec{E}=-\nabla \phi
$$

In practice, the microscopy results in an image of the voltage of $n$ by $m$ pixels. In this case, the electric field can be calculated using

$$
\begin{aligned}
& E_{x}\left(n+\frac{1}{2}, m\right)=-\phi(n, m)+\phi(n+1, m) \\
& E_{y}\left(n, m+\frac{1}{2}\right)=-\phi(n, m)+\phi(n, m+1)
\end{aligned}
$$

The voltage measured on the sample will be normalized by dividing it by the current that goes through the sample. This results in a normalized voltage which is equivalent to the 4-wire resistance between the ball and terminal to which $L_{\text {pot }}$ (see subsection III-C and Figure 4) is connected.

\section{MeAsurements}

\section{A. Sample fabrication}

The method is tested on a single layer meander pattern printed on a Diabase H-series 3D printer using the settings in Table I on top of a piece of Delrin which was sanded to increase adhesion. The gcode used for the print was generated using a custom slicing script. The print was intentionally under-extruded in order to create large non-uniformity in the conduction. An image of the resulting sample can be found in Figure 1. Silver epoxy (Chemtronics CW2400) is used to connect the sample to copper taper, onto which wires going to the measurement electronics are soldered. A strain relief is added by attaching the wires using cable shoes to the Delrin plate. 


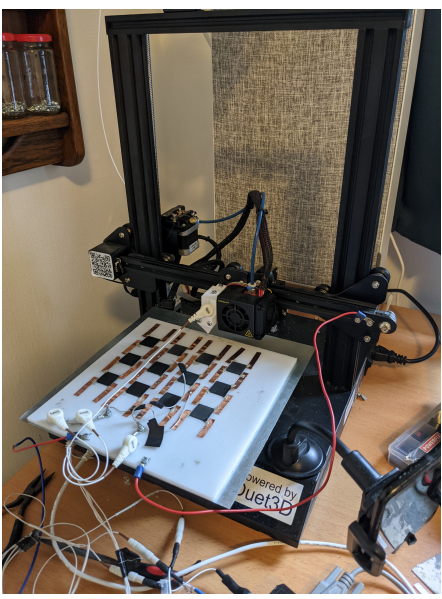

Fig. 2. A schematic image of the used measurement setup

TABLE I. PRINTING PARAMETERS

\begin{tabular}{c|c} 
Parameter & Value \\
\hline Layer height & $0.2 \mathrm{~mm}$ \\
Nozzle size & $0.4 \mathrm{~mm}$ \\
Line width & $0.4 \mathrm{~mm}$ \\
Line distance & $0.49 \mathrm{~mm}$
\end{tabular}

\section{B. Measurement setup}

To perform the measurement the Delrin plate with the 3D printed sample is placed on the bed of a Creality CR-20 3D printer, see Figure 2. A Mill-Max Omniball ball-head pogo pin with type number 7949-0-15-20-09-14-11-0 is mounted on the $x$ carriage of the 3D printed. During a measurement the probe is moved over the sample in a raster scan with a constant velocity of $4 \mathrm{~mm} \mathrm{~s}^{-1}$, see Figure 1 and Figure 3 The distance between the scan lines in the $y$-direction is $0.5 \mathrm{~mm}$. The motherboard of the 3D printer has been replaced by a Duet Maestro, which is controlled over USB with a Python script. This script moves the printer in the desired pattern and records the position to which it commands the printer to move. M400 commands were applied subsequent to every move to guarantee the Gl commands have finished, after which a timestamp is saved.

\section{Electronics}

The measurement electronics that are used consist of an auto-balancing bridge circuit called TiepieLCR which was previously developed by Schouten et al. [10] and of which a simplified schematic is shown in Figure 4 Both $V_{\text {out }}$ and $I_{\text {out }}$ are measured using a TiePie Engineering HS5-540 streaming 16-bit samples at $3.125 \mathrm{MS} \mathrm{s}^{-1}$. The voltage applied to $V_{\text {in }}$ is

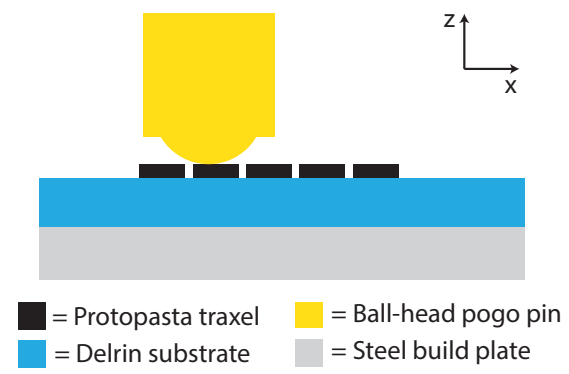

Fig. 3. A schematic image of the used measurement setup

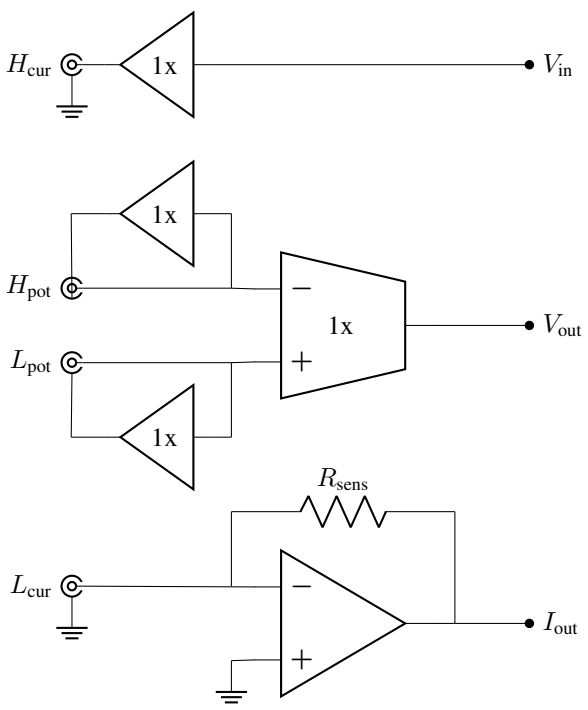

Fig. 4. Simplified circuit of the TiePieLCR [10].

generated using the arbitrary waveform generator of the HS5 and consists of a multi-sine with 7 different frequencies all with an amplitude of $86 \mathrm{mV}$ and a DC component with an amplitude of $200 \mathrm{mV}$. The current and the voltage at different frequencies are demodulated in real-time on a NVIDIA Quadro T1000 using a Fast Fourier Transform (FFT) based demodulation algorithm written in Python using the cupy library. $L_{\text {cur }}$, $L_{\text {pot }}$ and $H_{\text {cur }}$ are connected to the sample as shown in Figure 1 $H_{\text {pot }}$ is connected to the ball-head pogo pin on the $x$ carriage. The ground of the TiepieLCR is connected to the steel build plate and the frame of the $3 \mathrm{D}$ printer.

\section{Data processing}

The recorded position data and impedance data are synchronized using the recorded timestamps. After this, the LCR measurements were still delayed by $0.18 \mathrm{~s}$ compared to the position measurement. This delay was subtracted manually. The resulting data is re-sampled to a 200 by 200 grid, such that each pixel corresponds to 0.1 by $0.1 \mathrm{~mm}$. The resulting image was filtered by performing a $2 \mathrm{D}$ convolution with a rectangular window of size of 10 in the $y$ and 1 in the $x$ direction. 10 pixels in the image corresponds to the length of 2 scan lines and therefore this filter removes the noise due to the small differences between moving from left to right and from right to left. This results in a resolution of $0.1 \mathrm{~mm}$ in the $x$ direction and $1 \mathrm{~mm}$ in the $y$ direction.

\section{RESULTS}

\section{A. Measured Potential}

5(a) and 5(b) show respectively the real and the imaginary part of the normalized potential. It can clearly be seen that the real part of the potential is minimum at the bottom left corner and is maximum at the top right, where the connections are. For higher frequencies starting at $100 \mathrm{kHz}$ the imaginary part of the normalized potential starts to be significant. 


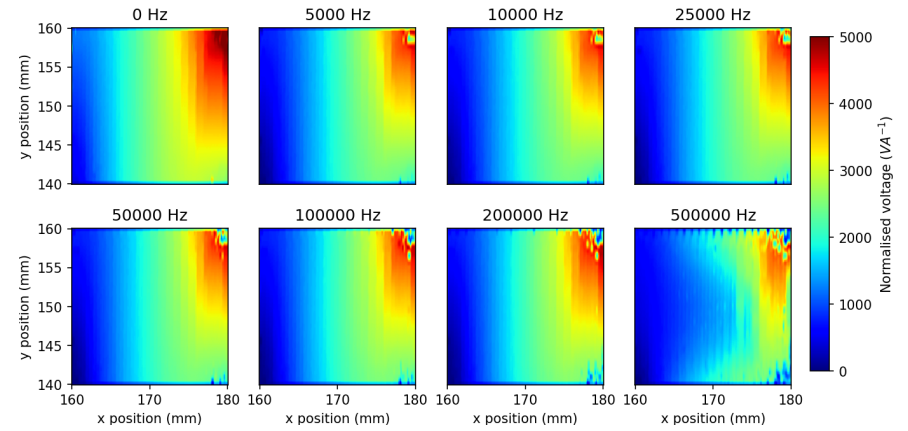

(a) Real part

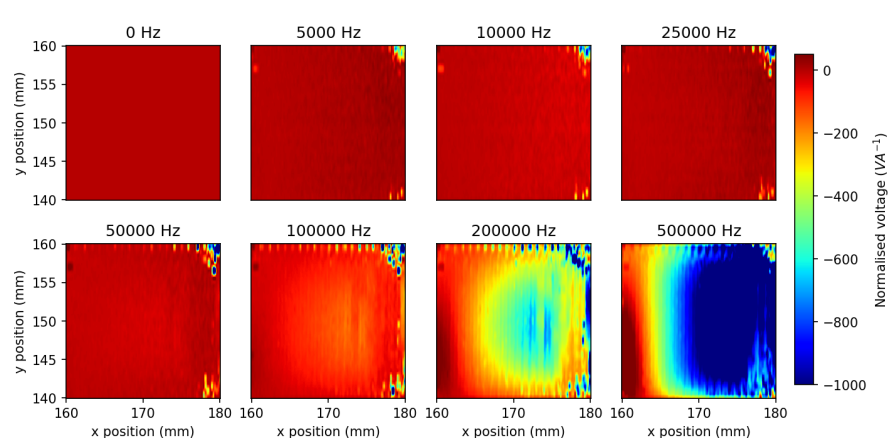

(b) Imaginary part

Fig. 5. The real and imaginary part of the normalized potential

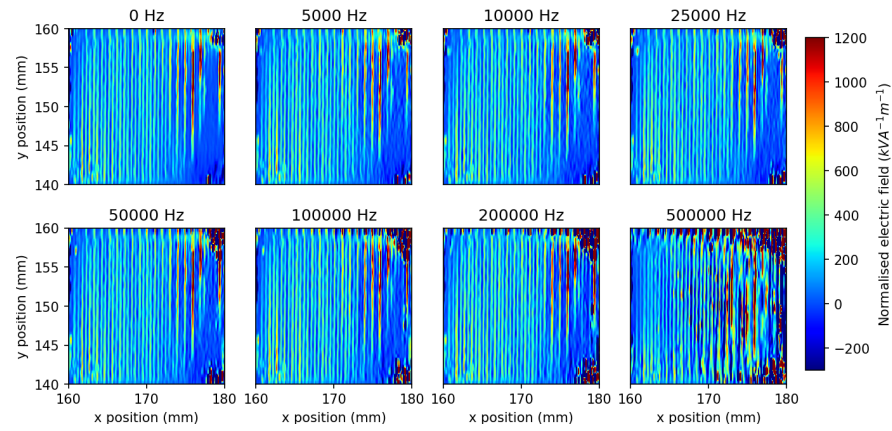

(a) The real part in the $x$-direction
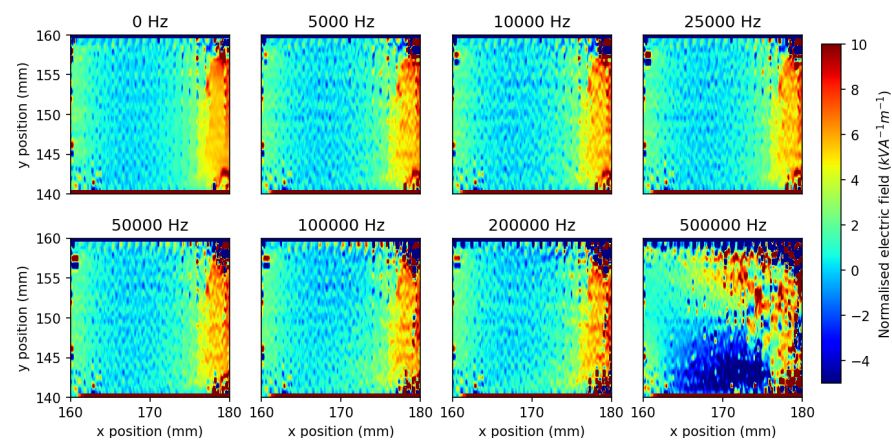

(c) The imaginary part in the $x$-direction
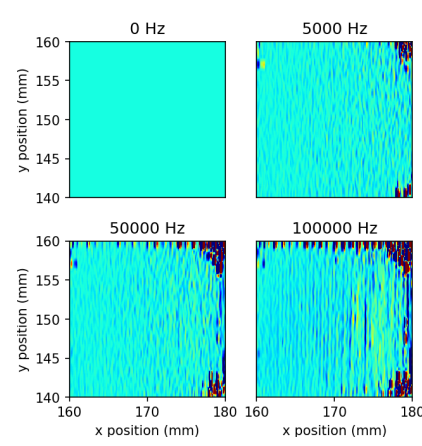

$100000 \mathrm{~Hz}$

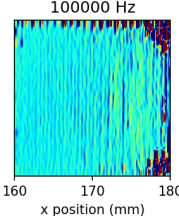

(b) The real part in the $y$-direction
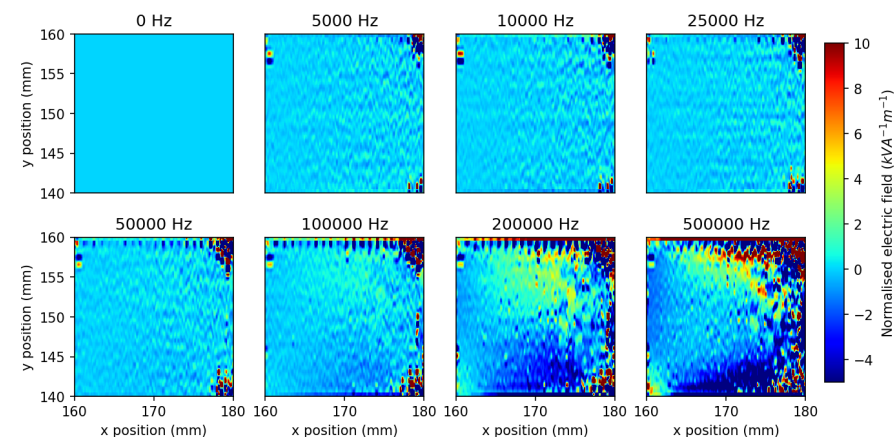

(d) The imaginary part in the $y$-direction

Fig. 6. The real and imaginary part of the normalized electric field in the $x$-and the $y$-direction

\section{B. Electric field}

The real part of the electric field in the $x$ - and $y$-direction can be found in respectively 6(a) and 6(b). The electric field in the $x$-direction clearly shows fluctuations at an interval that corresponds with the line distance. The real part of the electric field in the $y$ direction is more evenly distributed for lower frequencies. For higher frequencies the electric field in the $y$ direction also shows variations that correspond with the line distance.

The imaginary part of the electric field in the $x$ - and $y$ direction can be found in respectively 6(c) and 6(d) The imaginary part of the electric field in both directions is small when compared to the real part. However it can be seen that the imaginary part of the impedance in the $x$ direction is larger for all frequencies on the right side of the sample.

\section{DISCUSSION AND CONCLUSIONS}

We have shown that using a method similar to scanning impedance microscopy it is possible to quantitatively measure the real and the imaginary part of the in-plane electric field distribution inside a 3D printed conductor at different frequencies. This makes it possible to see local defects in the 3D printed conductor, such as improperly connected tracks of filament.

The results presented in this work only show the electric field inside the samples. To improve the conduction of the $3 \mathrm{D}$ printed sheet the anisotropic conductivity might be more interesting. To determine this conductivity the technique might be combined with an inverse finite element method simulation.

Currently, the scanning probe prevents the printer from being used for printing due to height constraints. However, when placed on a 3D printer with tool changing capability as a separate tool, it can be used to measure the potential in between deposition of subsequent layers, allowing for a more $3 \mathrm{D}$ view of the electric field inside the $3 \mathrm{D}$ printed structures. 


\section{REFERENCES}

[1] C. Weller, R. Kleer, and F. T. Piller, "Economic implications of 3D printing: Market structure models in light of additive manufacturing revisited," International Journal of Production Economics, vol. 164, pp. 43-56, 2015. [Online]. Available: http://dx.doi.org/10.1016/j.ijpe. 2015.02.020

[2] M. Schouten, G. Wolterink, A. Dijkshoorn, D. Kosmas, S. Stramigioli, and G. Krijnen, "A review of extrusion-based $3 d$ printing for the fabrication of electro- and biomechanical sensors," IEEE Sensors Journal, vol. 21, no. 11, pp. 12900-12912, 2021.

[3] A. Dijkshoorn, M. Schouten, S. Stramigioli, and G. Krijnen, "Modelling of anisotropic electrical conduction in layered structures 3d-printed with fused deposition modelling," Sensors (Switzerland), vol. 21, no. 11, May 2021.

[4] J. Zhang, B. Yang, F. Fu, F. You, X. Dong, and M. Dai, "Resistivity and its anisotropy characterization of 3d-printed acrylonitrile butadiene styrene copolymer (abs)/carbon black (cb) composites," Applied Sciences, vol. 7, no. 1, 2017. [Online]. Available: https://www.mdpi. com/2076-3417/7/1/20

[5] A. Dijkshoorn, M. Schouten, G. Wolterink, R. Sanders, S. Stramigioli, and G. Krijnen, "Characterizing the electrical properties of anisotropic, 3d-printed conductive sheets for sensor applications," IEEE Sensors Journal, vol. 20, no. 23, pp. 14218-14227, 2020.

[6] H. Watschke, K. Hilbig, and T. Vietor, "Design and characterization of electrically conductive structures additively manufactured by material extrusion," Applied Sciences, vol. 9, no. 4, 2019. [Online]. Available: https://www.mdpi.com/2076-3417/9/4/779

[7] S. V. Kalinin and D. Bonnell, "Scanning impedance microscopy: From impedance spectra to impedance images," MRS Online Proceedings Library 699, vol. 12, 2001.

[8] T. A. Hope and S. E. Iles, "Technology review: The use of electrical impedance scanning in the detection of breast cancer," Breast Cancer Research, vol. 6, no. 2, pp. 69-74, 2004.

[9] R. P. Feynman, R. B. Leighton, and M. Sands, The Feynman lectures on physics; New millennium ed. California Institute of Technology, 2010, originally published 1963-1965. [Online]. Available: https://www.feynmanlectures.caltech.edu/II_06.html

[10] M. Schouten, P. Patel, R. Sanders, and G. Krijnen, "3D printed differential force and position sensor based on lossy transmission lines," Transducers 2021, 2021. 